\title{
Design and simulation of muon ionization cooling channels for the Fermilab Neutrino Factory feasibility study
}

\author{
J. Monroe, P. Spentzouris, V. Balbekov, and P. Lebrun \\ Fermi National Accelerator Laboratory, Batavia, Illinois 60510 \\ G. Penn, C. Kim,* and E.S. Kim \\ Lawrence Berkeley National Laboratory, Berkeley, California 94707 \\ D. M. Kaplan \\ Illinois Institute of Technology, Chicago, Illinois 60621 \\ (Received 2 January 2001; published 17 April 2001)
}

\begin{abstract}
In the past few years, the concept of a high intensity muon storage ring has been pursued as an option for the next generation neutrino source. To produce the high intensity muon beam needed for the successful operation of a neutrino source, on the order of $10^{20}$ muon decays per year, the phase space occupied by the muon beam must be significantly reduced before the beam is accelerated. The initial transverse emittance of the muon beam before acceleration is assumed to be $9 \pi \mathrm{mm} \mathrm{rad}$. Because of the time limitation imposed by the muon lifetime, the technique employed to accomplish the desired emittance reduction is ionization cooling. In this paper we present two ionization cooling lattice designs, which use solenoidal focusing elements and liquid hydrogen absorbers to reduce the muon beam phase space. We discuss the design concepts and engineering constraints for these lattices and present simulation results obtained using a detailed tracing code with a complete model of muon-matter interactions. The reduction in transverse emittance is approximately a factor of 5. This result is within a factor of 2 of the total cooling requirements for a successful neutrino factory design and within a factor of 1.4 of the requirements for the main cooling section specified in the conceptual design of this machine.
\end{abstract}

DOI: 10.1103/PhysRevSTAB.4.041301

PACS numbers: 41.75.Lx

\section{INTRODUCTION}

The successful design of a high intensity neutrino source requires that the transverse phase space occupied by the muon beam after production, capture, and bunching be reduced by approximately a factor of 10 in each plane before the beam is accelerated. The technique which could accomplish the required cooling within the time limit imposed by the muon lifetime is ionization cooling [1-3]. In ionization cooling, the beam loses both transverse and longitudinal momentum by ionization energy loss, while passing through material. The longitudinal momentum is then restored to the beam in accelerating rf cavities. This sequence, repeated many times, results in a reduction of the angular spread of the beam particles, and thereby reduces the normalized transverse emittance.

Ionization cooling is limited by heating of the beam due to multiple Coulomb scattering in the energy-absorbing material. To minimize this heating effect, the absorbers are placed in a strong focusing field. An approximate differential equation for the rate of change of the normalized transverse emittance $\epsilon_{n}$ inside the absorber is [2,3]

$$
\frac{d \epsilon_{n}}{d z}=-\frac{1}{\beta^{2}} \frac{d E_{\mu}}{d z} \frac{\epsilon_{n}}{E_{\mu}}+\beta \gamma \frac{\beta_{\perp}}{2} \frac{d}{d z}\left\langle\theta^{2}\right\rangle,
$$

\footnotetext{
*Deceased
}

where $z$ is the path length, $E_{\mu}$ is the beam energy, $\beta=$ $v / c,\left\langle\theta^{2}\right\rangle$ is the mean-square planar scattering angle, and $\beta_{\perp}$ is the betatron function of the beam, with the beam size given by $\sigma_{x}=\sigma_{y}=\sqrt{\epsilon_{n} \beta_{\perp} / \beta \gamma}$. To first order, $\left\langle\theta^{2}\right\rangle=$ $\left(\frac{0.0136 \mathrm{GeV}}{p \beta c}\right)^{2} \frac{z}{L_{R}}$, where $L_{R}$ is the radiation length of the energy absorbing medium. Using this expression we can rewrite Eq. (1.1) as

$$
\frac{d \epsilon_{n}}{d z}=-\frac{1}{\beta^{2}} \frac{d E_{\mu}}{d z} \frac{\epsilon_{n}}{E_{\mu}}+\frac{1}{\beta^{3}} \frac{\beta_{\perp}(0.0136 \mathrm{GeV})^{2}}{2 E_{\mu} m_{\mu} L_{R} c^{2}} .
$$

Since the heating term is proportional to $\beta_{\perp}$ and inversely proportional to $L_{R}$, we place the absorbers in low $\beta_{\perp}$ regions and use material with high $L_{R}$ (liquid hydrogen) in order to maximize cooling.

\section{A. Cooling channel design considerations}

To obtain the strong beam focusing at the absorber needed for optimal cooling, several lattice configurations have been considered. Focusing by solenoids has been selected based on the results of design studies and on the constraints imposed for a realistic, technically feasible cooling channel design. Solenoidal focusing has the advantage of naturally focusing in both transverse planes, simplifying the design of the transverse beam optics. Solenoids can be used to focus a large transverse emittance beam to small $\beta_{\perp}$ at absorbers located outside the magnet or 
to provide continuous focusing, allowing the use of long absorbers inside the solenoid. A complication with the use of solenoids stems from the fact that a beam entering the magnet acquires angular momentum by its interaction with the radial component of the fringe field. In the absence of absorbers, this effect is reversible, since at the exit of the solenoid the beam loses the angular momentum acquired at the entrance. However, with an absorber inside the solenoid, the beam loses angular momentum as the transverse momentum decreases, so at the exit the cancellation is not exact and the beam retains a net angular momentum. If this net angular momentum is not compensated, it results in transverse emittance dilution outside of the focusing field.

The technique we use to compensate for the accumulation of angular momentum is to alternate the sign of the solenoidal field. In an ideal case, where this "field flip" is a step function, the solution would be to alternate the sign to cancel the residual angular momentum after each absorber. However, the field flip cannot be instantaneous, and its finite length induces a perturbation of the transverse motion and hence the longitudinal velocity of the beam particles. This perturbation can significantly dilute the beam phase space and introduces a coupling of the longitudinal and transverse motions.

Even without a field flip, there are several other effects peculiar to particle motion in ionization cooling channels with solenoids which cause transverse-longitudinal coupling. First is the dependence of path length on transverse momentum within a solenoidal field: particles at large transverse amplitude $A_{t}$ traverse a greater distance, therefore the time of flight of these particles is larger than that of particles with small $A_{t}$, with $A_{t} \sim$ $\sqrt{\left(r^{2} / \beta_{\perp}^{2}\right)+\left(p_{x} / p_{z}\right)^{2}+\left(p_{y} / p_{z}\right)^{2}}$. (Here $r$ is the distance from the beam axis, and a Cartesian right-handed coordinate system is used, with the $z$ axis coinciding with the axis of the solenoid. ${ }^{1}$ ) An off-axis particle has a longer time of flight than an on-axis particle with the same energy, and so will arrive in an rf cavity later and see a different accelerating gradient. Second, the presence of an absorber within the solenoidal field correlates energy loss with $A_{t}$, since particles at large $A_{t}$ traverse more material, thus losing more energy. The result of these two effects is a growth of the momentum spread of the beam which is correlated with $A_{t}$. In addition, energy loss in the absorber is momentum dependent, and the slope of the energy loss, $d E / d z$, as a function of energy is unfavorable at a beam momentum of $200 \mathrm{MeV} / c$, the mean beam momentum we have considered in our studies. This low momentum, $200 \mathrm{MeV} / c$ with a $10 \%$ spread, also causes some bunch distortion due to the nonrelativistic motion of the beam

\footnotetext{
${ }^{1}$ Unless explicitly stated otherwise, this coordinate system is used in all of the discussions in this paper.
}

particles. On the other hand, low momentum is favored for optimal cooling performance [see Eq. (1.1)], so the value of $200 \mathrm{MeV} / c$ is selected in order to maintain cooling without maximizing the undesirable effects presented above.

Neglecting any longitudinal-transverse coupling, for a fixed minimum betatron function $\beta_{\perp \text {,min }}$, the transverse emittance settles to an equilibrium value, beyond which any additional cooling is canceled by reheating caused by multiple Coulomb scattering [see Eq. (1.1)]. For the lattices presented here, after this equilibrium is achieved the transverse emittance increases with channel length with a rate which depends on the specifics of the lattice design. For example, the growth of the energy spread due to the energy-loss fluctuations in the interactions of the beam with the absorber (energy straggling) could cause crossing of energy resonances for some fraction of the beam in designs using periodic lattices. This effect could contribute significantly to emittance heating even prior to the point of equilibrium emittance.

The growth of the transverse amplitude-path-length coupling can be suppressed by frequently changing the sign of the magnetic field. In order to disrupt coherent Larmor motion, the length scale for the change in the magnetic field sign has to be shorter than the Larmor wavelength (see Sec. IC 1). As a result, the frequency with which we alternate the sign of the field is a subject of optimization for all cooling channel designs.

\section{B. Cooling channel lattices}

Several different lattices based on the use of short alternating solenoids have been considered for muon-beam cooling [4,5]. For a neutrino factory, the LBL group proposed a one-harmonic sinusoidal focusing field configuration (FOFO) [6], while Derbenev and Balbekov proposed a lattice featuring a long, constant solenoidal field of one polarity followed by another of opposite polarity, with only one field flip (single flip) [7].

Of these, the FOFO is conceptually the most straightforward and has been simulated in great detail with two distinct tracking codes, ICOOL [8] and DPGEANT [9], both of which include a complete description of the beam interactions with the absorbers. In this lattice, the field varies sinusoidally with a $2.2 \mathrm{~m}$ period, constraining the placement of absorbers and rf cavities. For example, the absorbers are placed at the points of $\beta_{\perp, \min }$ to minimize emittance growth due to multiple Coulomb scattering. The strict periodicity of the lattice, with 50-75 cells in the cooling channel, produces several betatron motion resonances which could be a significant source of particle loss.

The single flip cooling channel is the more technically straightforward of the two lattices; however, the longitudinal behavior of the muon beam in the field flip region is quite complex. There is no field periodicity in this lattice; the field on axis is constant and changes polarity only 
once in $150 \mathrm{~m}$. For this reason, there are no constraints on the placement of rf cavities or absorbers. The lack of periodicity eliminates the problem of betatron resonances as well; however, at the field flip the longitudinal-transverse correlations significantly dilute the beam phase space, accounting for the majority of particle loss in this design.

\section{Particle motion in solenoidal magnetic fields with absorbers}

Here we present a first-order treatment of particle motion in a lattice containing solenoids and absorbers. We consider two solutions to the problems of angular momentum growth and longitudinal-transverse-coupling-induced emittance dilution. In the alternating solenoid solution (FOFO), the field changes sign every few meters. In the long solenoid approach (single flip), the field changes sign only once.

Detailed discussions of particle motion in axial fields can be found in many references in the literature (see, for example, [10]). Here we present only the concepts relevant to the cooling channel design. In a uniform magnetic field $B_{z}$, without absorbers, there are three constants of motion: the magnitude of the transverse momentum and the two transverse components of the position of the Larmor center:

$$
\begin{gathered}
p_{t}=\sqrt{p_{x}^{2}+p_{y}^{2}}, \quad x_{L}=x+\frac{c p_{y}}{e B}, \\
y_{L}=y-\frac{c p_{x}}{e B} .
\end{gathered}
$$

Any distribution $F\left(x_{L}, y_{L}, p_{t}\right)$ is stationary, i.e., $(\delta F / \delta z)=0$, but here we will consider only axially symmetric beams, such as

$$
\frac{d^{2} N}{p_{t} d p_{t} r_{L} d r_{L}}=\frac{1}{4 \pi^{2} \sigma_{r_{L}}^{2} \sigma_{p_{t}}^{2}} \exp \left(-\frac{r_{L}^{2}}{2 \sigma_{r_{L}}^{2}}-\frac{p_{t}^{2}}{2 \sigma_{p_{t}}^{2}}\right)
$$

where $r_{L}=\sqrt{x_{L}^{2}+y_{L}^{2}}$ is the Larmor radius. In this case, the emittance of the beam for any transverse direction can be defined to be $\epsilon=\sigma_{r_{L}} \sigma_{p_{t}}$.

With the addition of energy loss and rf acceleration, the quasistationary distribution has the same form as Eq. (1.4). We can express the beam moments in terms of the initial beam properties, $\sigma_{r_{L}}^{0}$ and $\sigma_{p_{T}}^{0}$, and the scaled longitudinal coordinate $\zeta=\frac{z}{p}\left|\frac{d p}{d z}\right|[11]$, as

$$
\begin{aligned}
\sigma_{r_{L}}^{2} & =\left(\sigma_{r_{L}}^{0}\right)^{2}+\frac{\sigma_{x}^{* 2} \zeta}{2} \\
\sigma_{p_{t}}^{2} & =\left(\sigma_{p_{t}}^{0}\right)^{2} e^{-2 \zeta}+\sigma_{p_{x}}^{* 2}\left(1-e^{-2 \zeta}\right) .
\end{aligned}
$$

The terms above marked by a star are the ultimate equilibrium sizes of the beam in a perfect alternating solenoid channel [12]. Using Eq. (1.1) for a beam in equilibrium $\left(\frac{d \epsilon_{n}}{d z}=0\right)$, with an instantaneous field flip and $\left|B_{z}\right|=$ const, we can express the equilibrium beam moments as

$$
\begin{aligned}
& \sigma_{x}^{* 2}=\frac{\beta_{\perp}^{2}}{2} p \beta^{2} \frac{d\left\langle\theta^{2}\right\rangle}{d z}\left|\frac{d z}{d p}\right|, \\
& \sigma_{p_{x}}^{* 2}=\frac{p^{3}}{2} \frac{d\left\langle\theta^{2}\right\rangle}{d z}\left|\frac{d z}{d p}\right|,
\end{aligned}
$$

where $p$ is the total momentum, $|d p / d z|$ is the rate of momentum loss in the absorber, and $\theta$ is the planar scattering angle due to multiple Coulomb scattering. ${ }^{2}$

The longitudinal motion presents a very serious problem during cooling. As mentioned in Sec. I A, both the dependence of energy loss on momentum in an absorber and energy straggling increase the energy spread of the beam. Another serious effect is the dependence of longitudinal velocity on transverse momentum. In the FOFO we introduce an energy-amplitude correlation in the input beam to correct for this effect. In the single flip this effect is much smaller than the perturbation due to the field flip; therefore, we do not introduce such a correction. Any field flip introduces a longitudinal momentum spread, which results in an excitation of synchrotron oscillations with amplitude $E_{\mu}-E_{\text {ref }}$. This is a direct consequence of the large change of the transverse momentum of the beam particles at the field flip. At a reference energy $E_{\text {ref }}$, the longitudinal velocity $v_{z}=\frac{c^{2} p_{z}}{E_{\text {ref }}}=\frac{c}{E_{\text {ref }}} \sqrt{E_{\text {ref }}^{2}-m^{2} c^{4}-p_{t}^{2} c^{2}}$ of a particle which has no transverse momentum $\left(p_{t}=0\right)$ differs from that of a particle with nonzero transverse momentum. Since longitudinal focusing depends on $v_{z}$, the central energy of the beam in the rf bucket, $E_{c}$, depends on transverse momentum as

$$
E_{c}=E_{\mathrm{ref}} \sqrt{1+\frac{p_{t}^{2}}{m^{2} c^{2}}}
$$

where the reference (ideal) particle has energy $E_{\text {ref }}$ and no transverse momentum. Since at a field flip the transverse momentum changes, this dependence results in an effective shift of the central energy of the beam which was captured in the rf bucket before the flip. This is a serious problem in the single flip lattice, resulting in the excitation of synchrotron oscillations. However, in the case of the FOFO, where the field flips with a period much shorter than the synchrotron period, the resulting disturbance of the synchrotron motion is a high-frequency, periodic function of time, where harmonics of the synchrotron frequency are strongly suppressed.

\section{Alternating solenoid solution: FOFO lattice}

The FOFO lattice is, in its ideal form, a channel where the solenoidal magnetic field on axis has the form $B_{z}=$ $B_{0} \sin (2 \pi z / L)$, where $L$ is the periodicity and $B_{0}$ is the

\footnotetext{
${ }^{2}$ It is assumed that energy loss in an absorber is exactly compensated by rf acceleration.
} 
peak magnetic field on axis. For the typical mode of operation, small deviations in the magnetic field profile are unimportant, but in the geometry described below the magnetic field is very close to being a pure sinusoid due to the fact that the aperture of the coils producing the field is comparable to the period $L$.

The single-particle motion in a FOFO channel without any absorbers or rf cavities can be written in the Larmor frame of the beam as

$$
x_{R}^{\prime \prime}+\kappa_{0}^{2} \sin ^{2}(2 \pi z / L) x_{R}=0,
$$

where $\kappa_{0}=e B_{0} / 2 p_{z}$ and $x_{R}$ is the transverse spatial coordinate. Here, since the longitudinal magnetic field is changing sign, the Larmor frame is oscillating between the extreme values $\pm \kappa_{0} L / 2 \pi \mathrm{rad}$, rather than rotating in a circle as it does in a uniform solenoid. This form of Hill's equation reduces to a special case of Mathieu's equation, and thus the regions of stable motion are determined by the properties of the first order Mathieu function. In particular, particle motion is stable for all $\kappa_{0} L<7.21$, with the boundary between stable and unstable motion corresponding to $\pi$ phase advance per half-period.

Within this operating regime, the beta function oscillates from a minimum in the zero magnetic field regions to a maximum at the peak magnetic field. The extremes of the beta function are approximately given as

$$
\beta_{\min } \simeq \frac{1.4 L}{\kappa_{0} L}\left[1-\left(\frac{\kappa_{0} L}{7.21}\right)^{2}\right]^{ \pm 1 / 2} .
$$

Thus, for a given required momentum acceptance, the minimum beta function can be significantly reduced only by shortening the period $L$. Because this moves coils with opposite polarity closer to each other, there is more cancellation of the field produced by each coil, requiring either more current per coil for a given magnetic field or a reduction in beam momentum to make the beam easier to focus. The trend toward higher current densities and stronger magnetic forces on the coils makes it extremely challenging to build channels whose minimum beta function is well below the radius of the aperture of the magnets.

The channel considered here operates with the parameters $B_{0} \simeq 3.4 \mathrm{~T}, p_{z}^{\text {ref }} \simeq 200 \mathrm{MeV} / c$ (the longitudinal momentum of the reference particle), and $L \simeq 2.2 \mathrm{~m}$ yielding $\kappa_{0} L \simeq 5.6$. The input beam momentum is higher than the momentum of the $\pi$ resonance, which for the above channel parameters occurs for $P_{z} \simeq 155 \mathrm{MeV} / c$, as shown in Fig. 1. Also shown in Fig. 1 is the location of the stop bands as a function of $P_{z}$ for this channel configuration; it is clear that the maximum relative momentum spread allowed is on the order of $\pm 22 \%$. The choice of the beam central momentum value is a compromise between, on one hand, minimizing the required rf for cooling and making beam focusing easier (lower momentum is preferable), and, on the other hand, avoiding longitudinal emittance growth and reducing the relative momentum spread of the beam (higher momentum is preferable). The cell length is

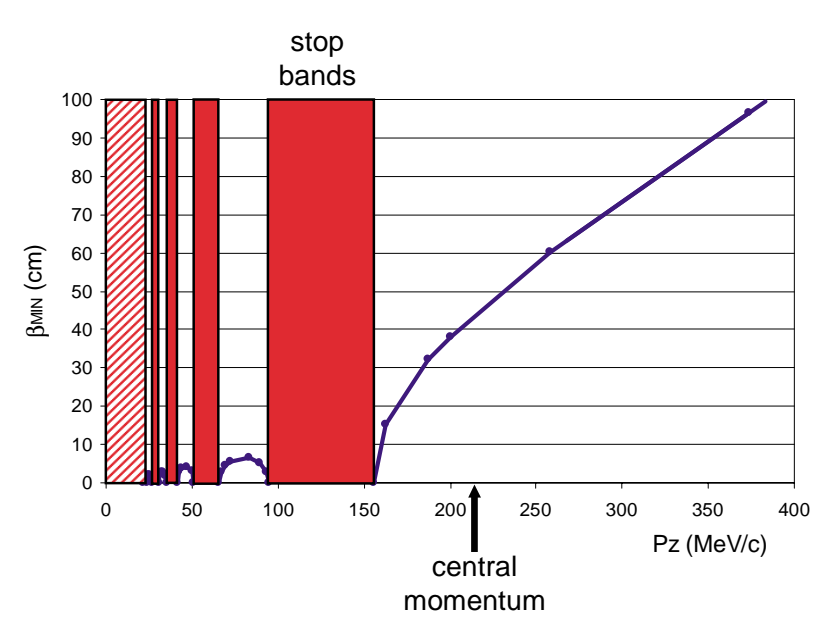

FIG. 1. (Color) Minimum $\beta$ function for the FOFO channel as a function of $P_{z}$.

limited by the magnetic aperture of $1.36 \mathrm{~m}$ diameter required to fit $200 \mathrm{MHz}$ rf cavities inside, which results in large cancellations of the field on axis due to fringe fields from neighboring coils; already at this period, the magnetic field increases from $3.4 \mathrm{~T}$ on axis to over $7 \mathrm{~T}$ at the coils.

The field period is less than the Larmor wavelength; therefore, there is no coherent Larmor motion in this alternating-solenoid-type cooling channel. As the reversal of field polarity changes the transverse momentum of the beam particles, the Larmor centers move, and the beam particles pick out new helical trajectories. This incoherence is illustrated in the projection of the motion onto the $x-z$ and $p_{x}-z$ planes in Figs. 2 and 3, respectively. With this field configuration, particles at large amplitudes do not necessarily remain at large amplitudes over more than one field period, which suppresses the transverse amplitude-path-length correlation.

In the full cooling channel, the matched beam has a minimum beta of $40 \mathrm{~cm}$ and a maximum of $80 \mathrm{~cm}$. These values are closer together than predicted from Eq. (1.9) for a monoenergetic beam, due to the large longitudinal

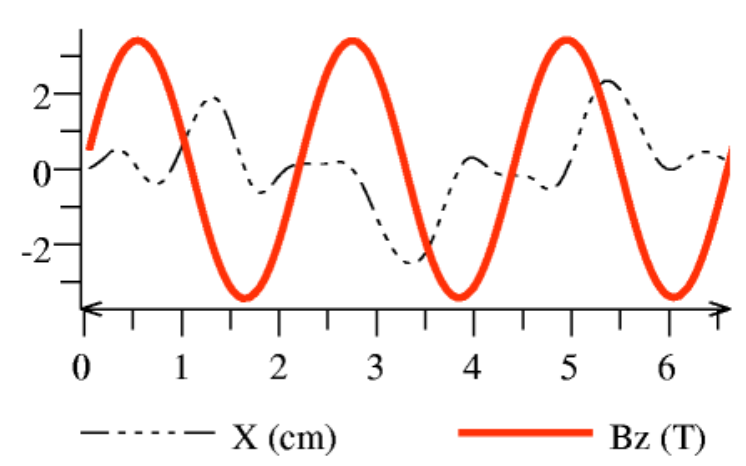

FIG. 2. (Color) $x(\mathrm{~cm})$ vs $z(\mathrm{~m})$ in three cells of the FOFO cooling channel. The dashed line is a projection of the reference particle's motion (note the incoherence of the Larmor rotation with respect to the magnetic field period). 


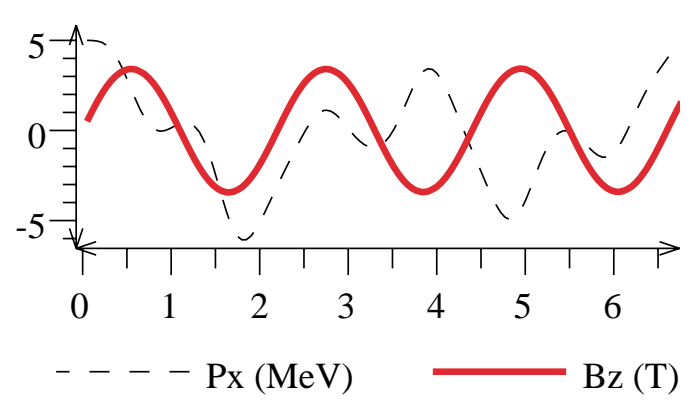

FIG. 3. (Color) $p_{x}(\mathrm{MeV} / c)$ vs $z(\mathrm{~m})$ in three cells of the FOFO cooling channel. The transverse momentum changes at the location of each field reversal, which gives rise to the incoherence of the Larmor motion, shown in the preceding figure.

and transverse emittances of the input beam. In addition, a correlation between the energy and the transverse amplitude of the initial particle distribution is necessary to match the beam properly into the rf system. This is the only non-Gaussian modification to the model beam distribution. The form of the correlation can be described in terms of the typical energy $E(A)$ for a particle with a given transverse amplitude $A, E(A)=E_{\mathrm{ref}}\left(1+A / A_{C}\right)$. Here, $E_{\text {ref }}$ is the nominal beam energy (corresponding to the reference particle for this design) and $A_{C}$ represents the intensity of the correlation, with small values indicating a large correlation. The (normalized) transverse amplitude $A$ can be written as

$$
A \equiv \frac{P_{z}}{m c \beta_{\perp}}\left(x^{2}+y^{2}\right)+\frac{\beta_{\perp} P_{z}}{m c}\left[\left(x^{\prime}\right)^{2}+\left(y^{\prime}\right)^{2}\right] .
$$

In this expression $\beta_{\perp}^{\prime}, B_{z}$, and the net canonical angular momentum were set to zero for simplicity. The value of $A_{C}$ chosen in this simulation for optimum performance is $340000 \pi \mathrm{mm} \mathrm{mrad}$. This correlation is sufficiently strong to push up the average energy of the initial beam by $10 \%$, increasing the energy spread as well. Without the correlation, beam transmission is reduced from $70 \%$ to $55 \%$, and the fraction of particles occupying the target phase space never goes above $35 \%$.

\section{Constant field solution: single flip lattice}

In the single field flip cooling channel, there is ideally no field periodicity, hence there is no betatron motion. Instead, the critical issue in the design of a single flip cooling channel is the control of the longitudinal motion.

To describe the beam dynamics in the single flip channel, let us first isolate the transverse motion by considering particle trajectories in a long solenoid passing through absorbers, without multiple Coulomb scattering. When the incoming beam is matched to the acceptance of the long solenoid, the beam particles follow helical trajectories about the field lines of the solenoid, with no change in the positions of the helix centers. As particles pass through an absorber their transverse momentum decreases, resulting in a smaller helix radius, given by $c p_{T} / e B$.
Even in the ideal case with no multiple Coulomb scattering effects, a long solenoidal channel with absorbers is by itself not sufficient for cooling, because such a configuration does nothing to reduce the transverse size of the beam. The beam at the start of the channel is rather large, $\sigma_{x} \simeq 4.5 \mathrm{~cm}$, which is unrealistic for the geometric acceptance of any downstream acceleration system. To reduce the transverse size of the beam, we introduce a second long solenoid with the opposite polarity.

The field between the two sections is reversed by a set of matching solenoids. This field reversal causes the Larmor centers to move closer to the axis of the second solenoid, where beam particles follow new helical trajectories with radii approximately proportional to the position of the Larmor center before the field flip. In the second solenoid, where the Larmor motion for all particles is centered approximately at the beam axis, cooling reduces both the transverse size and transverse momentum of the beam.

Multiple Coulomb scattering effects change this picture in the following way: the Larmor radius of the helical trajectories about the field lines cannot decay past the radius corresponding to the transverse momentum introduced by the scattering process and there is a diffusion in the radial distribution of the Larmor centers. Figure 4 illustrates an example of particle motion in such a channel. The random shifts in the position of a particle's Larmor center due to multiple Coulomb scattering lead to the somewhat wobbly trajectory in Fig. 4.

The value of the minimum achievable emittance differs for the first and second solenoids in the single flip cooling channel design, designated by $\epsilon_{1}^{\text {(out) }}$ and $\epsilon_{2}^{\text {(out) }}$ in the following. Using the notation from Sec. IC, in the first so-

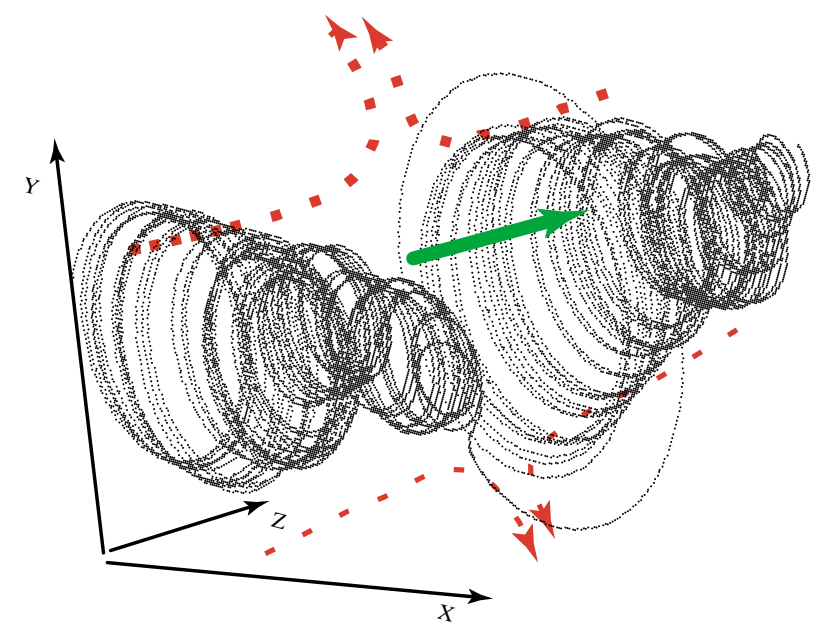

FIG. 4. (Color) $X, Y$ vs $z$ particle motion in the single flip channel. The dashed lines indicate magnetic field lines and the location of the change in field polarity. In the first half of the cooling channel the transverse momentum and therefore the radius of the Larmor motion decreases, but the distance of the Larmor center from the beam axis changes only due to multiple Coulomb scattering. In the second half, the Larmor center coincides with the beam axis, and the radius of the Larmor motion decreases with the transverse momentum. 
lenoid the input beam is much larger than the equilibrium emittance,

$$
\sigma_{r_{L 1}}^{0} \gg \sigma_{x}^{*}, \quad \sigma_{p_{t} 1}^{0} \gg \sigma_{p_{x}}^{*} .
$$

For the following calculations we will assume that the beam is matched to the acceptance of the solenoid and that the mean canonical angular momentum of the beam is zero. In this case, $\sigma_{r_{L 1}}^{0} / \sigma_{p_{t} 1}^{0} \simeq c / e B$; this condition is slightly violated when the muons are born in a magnetic field.

From Eq. (1.6), for matched beams, transverse emittance in a uniform solenoidal field tends to the same limit as in an alternating solenoidal field, but twice as fast. However, the behavior of the beam envelope in a uniform field is quite different from that in a periodic field. The beam radius decays with the transverse momentum in an alternating solenoidal field, but grows slowly in the uniform solenoid due to the diffusion of Larmor centers caused by multiple Coulomb scattering. As a result, the beam emittance,

$$
\begin{aligned}
\epsilon^{2} & =\sigma_{r_{L}}^{2} \sigma_{p_{t}}^{2} \\
& =\left[\left(\sigma_{r_{L}}^{0}\right)^{2}+\frac{\sigma_{x}^{* 2} \zeta}{2}\right]\left[\left(\sigma_{p_{t}}^{0}\right)^{2} e^{-2 \zeta}+\sigma_{p_{x}}^{* 2}\left(1-e^{-2 \zeta}\right)\right],
\end{aligned}
$$

decreases at the beginning and grows later having a minimum at some value of the scaled length $\zeta$ [12]. The cooling channel should end when this minimum is achieved. In the first solenoid, using the conditions from (1.11), we find that the minimum occurs at

$$
\chi_{1} \simeq \ln \frac{2 \sigma_{r_{L 1}}^{0} \sigma_{p_{t} 1}^{0}}{\sigma_{x}^{*} \sigma_{p_{x}}^{*}}=\ln \frac{2 \epsilon_{1}^{0}}{\epsilon^{*}},
$$

expressed in terms of the initial and equilibrium values of the transverse emittance. At the emittance minimum, the transverse momentum has decayed almost to equilibrium, while the increase in beam radius is relatively small, thus one can write for the first solenoid

$$
\begin{gathered}
\sigma_{r_{L 1}}^{\text {(out) }} \simeq \sigma_{r_{L 1}}^{0}, \quad \sigma_{p_{t} 1}^{\text {(out) }} \simeq \sigma_{p_{x}}^{*}, \\
\epsilon_{1}^{\text {(out) }}=\sigma_{r_{L 1}}^{\text {(out) }} \sigma_{p_{t} 1}^{\text {(out) }} \simeq \sqrt{\frac{\epsilon_{1}^{0} \epsilon^{*}}{2}} .
\end{gathered}
$$

Following the first long solenoid is a matching section in which the axial magnetic field flips from $B$ to $-B$. The radial magnetic field in this region changes the transverse momentum of all particles. If the change in axial field polarity occurs quickly enough, the particle's transverse spatial coordinates do not change, resulting in

$$
p_{x 2}^{0}=p_{x 1}^{\text {(out) }}-\frac{e B y}{c}, \quad p_{y 2}^{0}=p_{y 1}^{\text {(out) }}+\frac{e B x}{c} .
$$

The actual transverse drift in the matching section can be estimated as

$$
\frac{\Delta r}{r} \simeq \frac{e B L}{2 p c}
$$

where $L \simeq R$ is the effective length of the field flip and $R$ is the radius of the matching solenoid. Using Eq. (1.3), the following transformation occurs at the field flip:

$$
r_{L} \Longleftrightarrow \frac{p_{t} c}{e B} .
$$

After the field flip, the muon beam distribution can be described by relation (1.4) with the following initial conditions $[12,13]$ :

$$
\sigma_{r_{L 2}}^{0}=\frac{c \sigma_{p_{t} 1}^{\text {(out) }}}{e B} \simeq \frac{c \sigma_{p_{x}}^{*}}{e B} \simeq \frac{\sigma_{x}^{*}}{2}
$$

and

$$
\sigma_{p_{t} 2}^{0}=\frac{e B \sigma_{r_{L 1}}^{\text {(out) }}}{c} \simeq \frac{e B \sigma_{r_{L 1}}^{0}}{c} \simeq \sigma_{p_{t} 1}^{0} .
$$

Thus the field flip causes a strong decrease in the radius of Larmor centers, almost instantaneously. With initial conditions (1.18) and (1.19), the minimum emittance in the second solenoid is

$$
\epsilon_{2}^{\text {(out) }} \simeq \frac{\epsilon^{*}}{2}\left(\sqrt{2 \chi_{2}}+\frac{1}{\sqrt{2 \chi_{2}}}\right),
$$

where $\chi_{2} \simeq \frac{1}{2} \ln \left(\frac{2 \epsilon_{1}^{0}}{\epsilon^{*}} \ln \frac{2 \epsilon_{1}^{0}}{\epsilon^{*}}\right)$ is the value of $\zeta$ where the minimum emittance is achieved. Strictly speaking, in the calculation of the emittance minimum in the second solenoid, it is necessary to take into account the kick from the radial field at the exit of the solenoid, which increases the transverse momentum spread. However, this effect is not strong because the beam size here is relatively small.

The dominant cause of particle loss in a single field flip cooling channel is the sharp increase in momentum spread at the field flip. Following Eq. (1.7), with the exchange of Larmor radius for transverse momentum at the field flip, Eq. (1.17), the change of the central energy of the bucket after the field flip is described by

$$
E_{c} \simeq E_{\text {ref }} \sqrt{1+\frac{2 e B \epsilon_{1}^{0}}{m^{2} c^{2}}} .
$$

Since the excitation of synchrotron oscillations at this point is proportional $B$, the limiting factor in the performance of the single flip cooling channel is ultimately the field of $5 \mathrm{~T}$. The value of the field cannot be further reduced without losing significantly in transverse cooling performance.

\section{Design Goals}

An initial design study of all the components of a neutrino factory [14] concluded that the cooling needed is approximately 1 order of magnitude in each transverse plane, without unmanageable longitudinal dilution of the bunch. Given that the "minicooling" upstream of the cooling channel reduces transverse emittance by a factor of 
$\approx \sqrt{2}[14,15]$, the initial transverse emittance of the beam into the cooling channel was assumed to be $9 \pi \mathrm{mm} \mathrm{rad}$, and we targeted an output emittance of $1.5 \pi \mathrm{mm} \mathrm{rad}$. The input rms bunch length was $8.5 \mathrm{~cm}$, and the momentum spread of the beam was $10 \%$.

While rms emittance provides a useful gauge of cooling performance, for a neutrino factory the number of muons decaying in the downward straight section of a storage ring is the ultimate figure of merit. Thus, the most useful measure of cooling channel performance is the number of muons exiting the cooling channel within the acceptance of the acceleration system. This acceptance was specified as a four-dimensional hypersphere in transverse phase space with a radius equal to $2.5 \sigma$, where $\sigma^{2}=1.5 \pi \mathrm{mm} \mathrm{rad}$, and an ellipse in longitudinal phase space with an area of $150 \pi \mathrm{mm}$ [15]. Within these limits, $99 \%$ of the muons are expected to be accelerated and delivered to the storage ring, while a negligible fraction of muons lying outside these limits will be accelerated. In Secs. II B and III B we present the yield into this acceptance for the FOFO and single flip cooling channel designs, respectively.

\section{E. Design Constraints}

Engineering feasibility for superconducting solenoids is a complicated function that depends on such parameters as field, current density, and stress on the conductor. While a detailed engineering study has yet to be performed, a conservative rule of thumb for solenoids built from $\mathrm{Nb}_{3} \mathrm{Sn}$, based on keeping the hoop stress within manageable limits, is that $B J R<350 \mathrm{MPa}$ [16], where $B$ is the magnetic field at the coil, $R$ is the radius, and $J$ is the current density, all evaluated where their product is a maximum. Coils satisfying this inequality should have forces on the windings that are within acceptable limits.

The thickness of the absorber vessel windows is a critical parameter, from the standpoint of both technical feasibility and cooling performance. The windows must be thick enough to sustain the $\mathrm{LH}_{2}$ pressure, yet as thin as possible to minimize emittance heating due to multiple scattering. The window thicknesses for these cooling channel lattices and beams have been chosen based on the ASME standard for pressure vessels [17], which, for absorber radius of $\gtrsim 10 \mathrm{~cm}$ and $\mathrm{LH}_{2}$ pressure of $\approx 1 \mathrm{~atm}$, dictates a minimum window thickness of approximately $200 \mu \mathrm{m}$. Assuming aluminum-alloy windows, a $200 \mu \mathrm{m}$ window thickness is $0.2 \%$ of a radiation length. For comparison, $10-30 \mathrm{~cm}$ of liquid hydrogen is $1.2 \%-3.5 \%$ of a radiation length.

A $200 \mathrm{MeV} / c$ muon beam loses on average $3.6 \mathrm{MeV}$ of energy in a $12 \mathrm{~cm}$ long absorber. At the beginning of the cooling channel, where bunches of up to $10^{13}$ muons may be incident at a $15 \mathrm{~Hz}$ repetition rate, including power dissipated by decay electrons and $\mathrm{x}$ rays from the rf cavities, power of the order of $100 \mathrm{~W}$ will be dissipated in such an absorber. This power dissipation is within the range that has been handled successfully at the SLAC [18] and Bates [19] laboratories, utilizing a flow-through cooling loop design with an external heat exchanger.

The choice of parameters for the rf cavities is also an important factor in the cooling performance. The parameters used in our study follow technical feasibility guidelines for achievable gradients and frequencies defined in Ref. [15]. To maximize the accelerating gradient per unit rf power, the cavities are closed at the ends by thin beryllium windows [20]. The resulting trade-off in cooling performance between gradient and window-induced multiple scattering becomes a part of the design optimization.

The optimization - currently in progress - of these two types of cooling channels depends strongly on the application of realistic hardware design constraints. At this stage, both configurations have had preliminary engineering design reviews, and thus far meet the standards of technical feasibility.

\section{THE FOFO CHANNEL}

\section{A. Cooling channel description}

The FOFO cooling channel has two stages: the first is $44 \mathrm{~m}$ long consisting of 40 cells, the second is $110 \mathrm{~m}$ long consisting of 100 cells. The reduction in transverse beam size in the first cooling stage allows absorbers of smaller radius to be used in the second stage. The concomitant reduction in absorber window thickness reduces the multiple scattering per absorber, allowing the second stage to achieve smaller transverse emittance. Two absorber designs are used, one in each of the two cooling stages. The first is $15 \mathrm{~cm}$ in radius and $12.6 \mathrm{~cm}$ in length, enclosed by $400 \mu \mathrm{m}$ aluminum windows, and the second is $10 \mathrm{~cm}$ in radius and $13.2 \mathrm{~cm}$ in length, enclosed by $200 \mu \mathrm{m}$ aluminum windows. The two different absorber lengths are chosen to give the same energy loss $(3.9 \mathrm{MeV})$ per cell,

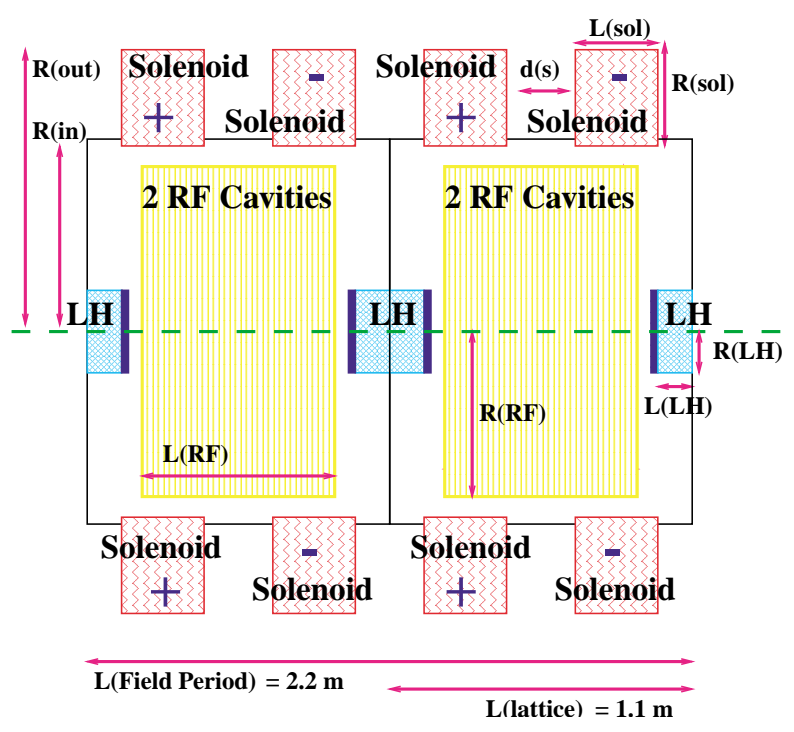

FIG. 5. (Color) FOFO cooling cell layout. 
TABLE I. List of parameters for the $3.4 \mathrm{~T}$ FOFO.

\begin{tabular}{lc}
\hline \multicolumn{1}{c}{ Parameters } & Values \\
\hline Length of section, $\Delta L$ & $1.1 \mathrm{~m}$ \\
Maximum magnetic field & $3.4 \mathrm{~T}$ \\
Number of sections per stage & $40 / 100$ \\
Length of hydrogen $\left(\mathrm{LH}_{2}\right)$ absorber & $0.126 / 0.132 \mathrm{~m}$ \\
Density of $\mathrm{LH}_{2}$ & $0.0708 \mathrm{~g} / \mathrm{cm}^{3}$ \\
Thickness of hydrogen windows $(\mathrm{Al})$ & $400 \mu \mathrm{m} / 200 \mu \mathrm{m}$ \\
Energy loss per section, nominal & $\approx 3.9 \mathrm{MeV}$ \\
Radial aperture, in LH & \\
Length of linac (per section) & $15.0 / 10.0 \mathrm{~cm}$ \\
Number of rf cells & $0.6586 \mathrm{~m}$ \\
Frequency (MHz) & 2 \\
Peak field, on axis & $201.25 \mathrm{MHz}$ \\
Optimum sync. phase $\phi_{s}$ & $15 \mathrm{MV} / \mathrm{m}$ \\
Acceleration at optimum $\phi_{s}$ & $29.7^{\circ}$ \\
Beryllium window thickness & $\approx 4.3 \mathrm{MV}$ \\
Radial aperture, linac & $125 \mu \mathrm{m}$ \\
Nominal momentum $P_{0}$ & $r=17 \mathrm{~cm}$ \\
Normalized transverse emittance $\epsilon_{T n}$ & $210 \mathrm{MeV} / c$ \\
$\beta_{\perp}$ & $9000 \pi \mathrm{mm} \mathrm{mrad}$ \\
$\sigma_{x}$, lab frame & $40.0 \mathrm{~cm}$ \\
$\sigma_{P t}$, lab frame & $4.5 \mathrm{~cm}$ \\
Longitudinal bunch spread $\sigma_{z}$ & $20.0 \mathrm{MeV} / c$ \\
Longitudinal emittance $\epsilon_{L n}$ & $10.0 \mathrm{~cm}$ \\
$6 \mathrm{D}$ invariant emittance & $15 \mathrm{~mm}$ \\
\hline \hline
\end{tabular}

such that the rf system does not require retuning to maintain an approximately constant beam energy.

The FOFO magnetic field is sinusoidal at the beam axis, with $1.1 \mathrm{~m}$ half-period and $3.4 \mathrm{~T}$ maximum field on axis. The magnetic field is generated by $30 \mathrm{~cm}$ long Helmholtz coils with an inner diameter of $1.36 \mathrm{~m}$ and outer diameter of $2.06 \mathrm{~m}$. Each half-period of the magnetic field has two coils of the same polarity separated by a $20 \mathrm{~cm}$ gap (for power coupling to the rf), and magnets in adjacent cells have opposite polarity. All coils have a current density of $49 \mathrm{~A} / \mathrm{mm}^{2}$. The large aperture is necessary to accommodate the 201.25 MHz rf cavities. While the peak field on axis is $3.4 \mathrm{~T}$, the peak field at the coils is close to $7 \mathrm{~T}$.

The layout of the FOFO cooling cell is shown in Fig. 5, and the channel parameters are tabulated in Table I. Each cooling cell consists of a half-period of the FOFO lattice, with two $\pi / 2$ mode pillbox rf cavities and a liquid hydrogen absorber. The rf cavities have a peak field of $15 \mathrm{MV} / \mathrm{m}$, use beryllium windows, and are each $32.93 \mathrm{~cm}$ long, giving an rf phase advance per cavity equal to $\pi / 2$. Each pair of rf cavities accelerates the beam by $4.3 \mathrm{MeV} / c$ at a synchronous phase of $29.7^{\circ}$. This energy is then removed by the hydrogen absorber. In this example, the channel is tuned so that there is no net acceleration of the beam.

\section{B. Simulation results}

Figure 6 shows results from a simulation of the FOFO channel. Within 6D phase space cuts of $150 \mathrm{~mm}$ longi-
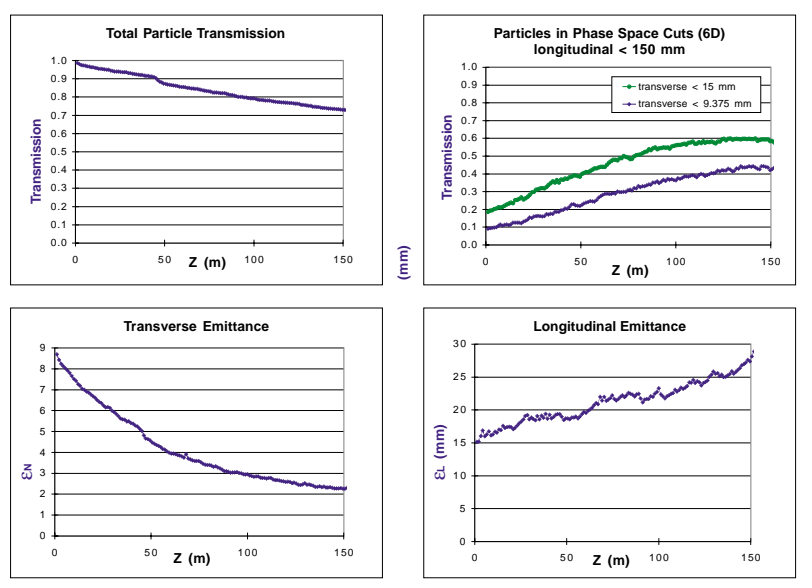

FIG. 6. (Color) FOFO channel performance as a function of the longitudinal coordinate $z$. Top right: fractional transmission; top left: transmission into the acceleration acceptance cuts, upper curve (blue line) with a $9.375 \mathrm{~mm}$ rad transverse cut, lower curve (green line) with a $15 \mathrm{~mm}$ rad transverse cut; bottom left: transverse emittance; bottom right: longitudinal emittance.

tudinal and $9375 \pi \mathrm{mm}$ mrad transverse emittances, the fraction of initial muons increases from $10 \%$ to $45 \%$. An additional $25 \%$ of the initial beam is transmitted to the end of the cooling channel but lies outside of the target region of phase space. The remaining $30 \%$ of the original beam is lost due to particle decays, scraping against apertures, or stopping in the absorbers. Of the lost muons, approximately $10 \%$ are lost due to muon decays, and about $6 \%$ because of muons with sufficiently high transverse amplitude to scrape against the $\mathrm{rf}$ windows (at $17 \mathrm{~cm}$ radius). In addition, $44 \mathrm{~m}$ into the channel, the liquid hydrogen absorber radius is reduced to $10 \mathrm{~cm}$, causing another $4 \%$ of the input beam to be scraped. The remaining losses are from a combination of discrete events with largeangle scattering or large energy loss, or particles falling out of the rf bucket and eventually crossing the $\pi$ resonance for the FOFO channel (at a momentum of $155 \mathrm{MeV} / c$ for this lattice), which causes them to fall outside the transverse acceptance of the channel.

In the FOFO channel, the beam is cooled in a selfsimilar manner, so that the transverse rms properties maintain their proportion. In the longitudinal direction, there is heating in phase space due to the unfavorable slope of the energy loss $d E / d z$ as a function of energy and the energy straggling. This leads approximately to a doubling of occupied longitudinal phase space, which results in reduced transmission rather than increased emittance, as particles fall out of the rf bucket.

The beta function of $40 \mathrm{~cm}$ at the liquid hydrogen absorbers corresponds roughly to an equilibrium emittance of $2200 \pi \mathrm{mm} \mathrm{mrad}$. The final emittance of the beam is very close to this value. These results are summarized in Table II. For comparison, a total energy of $\approx 600 \mathrm{MeV}$ was removed by the absorbers and returned via rf acceleration, 
TABLE II. Evolution of beam parameters in the FOFO cooling channel. The number of muons per proton incident on target is shown in parentheses in the last three rows.

\begin{tabular}{cccc}
\hline \hline Parameter & $z=0 \mathrm{~m}$ & $z=100 \mathrm{~m}$ & $z=150 \mathrm{~m}$ \\
\hline$\epsilon_{T}(\mathrm{~mm} \mathrm{rad})$ & 9.0 & 3.0 & 2.2 \\
$\epsilon_{L}(\mathrm{~mm})$ & 15 & 22 & 27 \\
$N_{9 \mathrm{~mm}}(\%)$ & 10 & 37 & $43(0.095)$ \\
$N_{15 \mathrm{~mm}}(\%)$ & 19 & 55 & $60(0.132)$ \\
$N_{\text {part }}(\%)$ & $100(0.22)$ & $80(0.176)$ & $73(0.16)$ \\
\hline \hline
\end{tabular}

which in the absence of multiple scatter would have cooled the beam from $9000 \pi \mathrm{mm}$ mrad to $\approx 450 \pi \mathrm{mm} \mathrm{mrad}$.

\section{THE SINGLE FLIP COOLING CHANNEL}

\section{A. Cooling channel description}

The single flip cooling channel has two stages. Between the two cooling stages there is a $2.47 \mathrm{~m}$ long matching section in which the axial field changes polarity. The first
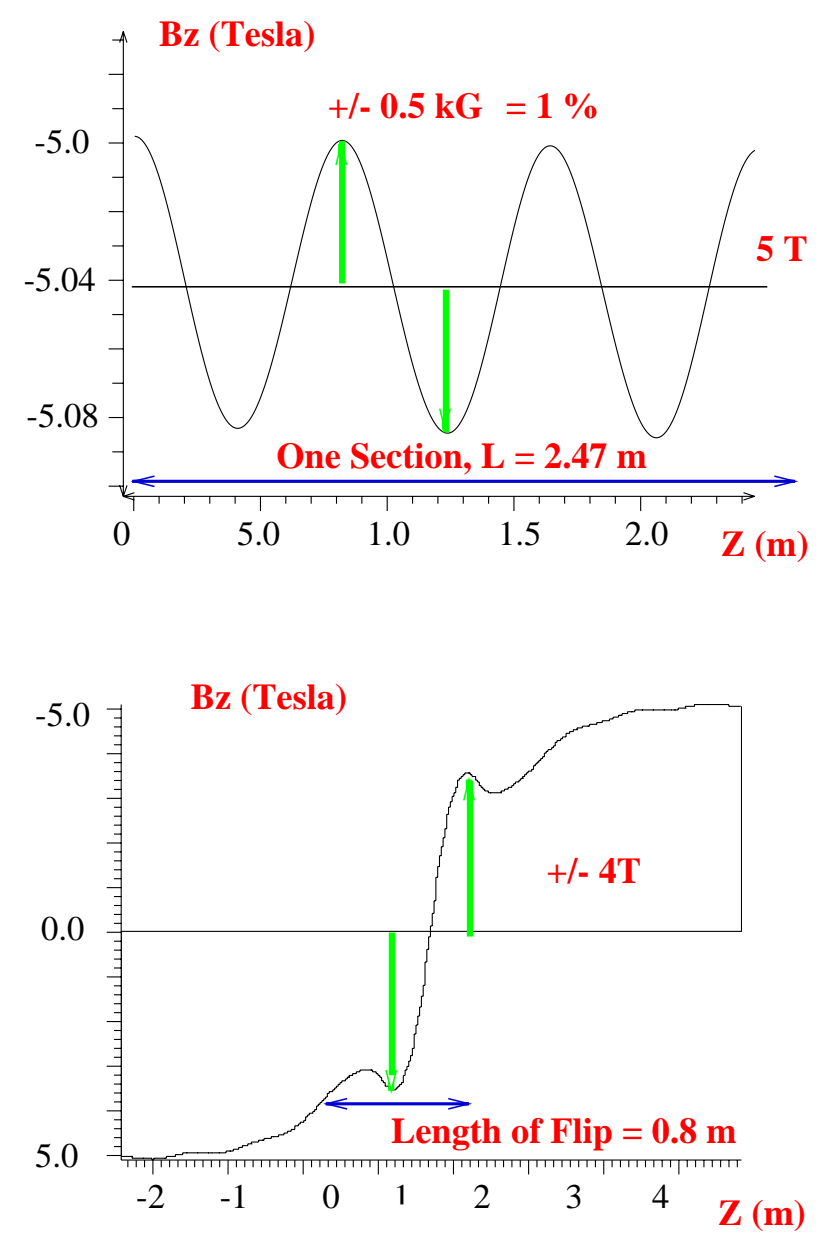

FIG. 7. (Color) Top: variation of the axial field $B z$ (kGauss) at $r=0 \mathrm{~cm}$ in the single flip cooling channel, for the constant field stages. Bottom: variation of the axial field at $r=0$ in the matching (field flip) section. stage consists of twenty-one $2.47 \mathrm{~m}$ long cooling cells, at a nearly constant magnetic field of $-5 \mathrm{~T}$ on axis. The second stage consists of forty-two $2.47 \mathrm{~m}$ long cells at $+5 \mathrm{~T}$ on axis. Within each cooling stage the magnetic fields vary by only $\pm 1 \%$; for this reason there is very little modulation of the beam envelope. The axial field versus $z$ for both cooling stages and the matching section is shown in Fig. 7. The beam envelope modulation has a wavelength of $\approx 1.5 \mathrm{~m}$, with an amplitude of $\pm 2 \mathrm{~cm}$. The Larmor radius of the nominal particle is $0.6 \mathrm{~cm}$, and the Larmor wavelength is $\approx 3 \mathrm{~m} \mathrm{~m}$.

The most sensitive parameter of this cooling channel design is the derivative with respect to $z$ of the $z$ component of the magnetic field at the location of the field flip. Ideally, when the field flip is very fast, there is no drift of the Larmor centers [Eq. (1.6) for $L \rightarrow 0$ ]. In this case the minimum transverse-longitudinal correlation is induced in the beam, since there is no drift of the Larmor centers and the $p_{t}$ change depends on the Larmor radius, and hence matching into the rf bucket acceptance produces maximal transmission. In a more realistic case, $p_{T}$ and thus the difference of the central energy of the beam in the rf bucket, $E_{c}$, to $E_{\text {ref }}$ increase significantly [see Eq. (1.21)] due to the radial field in the flip region, which excites a large synchrotron oscillation. As a result, the largest particle losses occur within 8 to $12 \mathrm{~m}$ after the field flip, although the synchrotron period in this channel is $\approx 28 \mathrm{~m}$.

TABLE III. List of parameters for the $5 \mathrm{~T}$ single flip cooling channel.

\begin{tabular}{lc}
\hline \hline \multicolumn{1}{c}{ Parameters } & Values \\
\hline Length of section, $\Delta L$ & $2.47 \mathrm{~m}$ \\
Maximum magnetic field & $5.0 \mathrm{~T}$ \\
Number of cells per stage & $21 / 42$ \\
Length of hydrogen $\left(\mathrm{LH}_{2}\right)$ absorber & $0.32-0.35 \mathrm{~m}$ \\
Density of LH $\mathrm{LH}_{2}$ & $0.0708 \mathrm{~g} / \mathrm{cm}^{3}$ \\
Thickness of hydrogen windows (Al) & $300 \mu \mathrm{m}$ \\
Energy loss per cell, nominal & $\approx 10 \mathrm{MeV}$ \\
Radial aperture, in LH $\mathrm{H}_{2}$ & $=20.0 \mathrm{~cm}$ \\
Length of linac (per section) & $1.974 \mathrm{~m}$ \\
Number of rf cells & 6 \\
Frequency (MHz) & $201.25 \mathrm{MHz}$ \\
Peak field, on axis & $15 \mathrm{MV} / \mathrm{m}$ \\
Optimum sync. phase $\phi_{s}$ & $28.65^{\circ}$ \\
Acceleration at optimum $\phi_{s}$ & $\approx 12.5 \mathrm{MV}$ \\
Beryllium window thickness & $125 \mu \mathrm{m}$ \\
Radial aperture, linac & $r=19 \mathrm{~cm}$ \\
Nominal momentum $P_{0}$ & $\rightarrow 280 \mathrm{MeV} / c$ \\
& $($ at the field flip) \\
Normalized transverse emittance $\epsilon_{T n}$ & $12250 \pi \mathrm{mm} \mathrm{mrad}$ \\
$\beta_{\perp}$ & $21.0 \mathrm{~cm}$ \\
$\sigma_{x}$, lab frame & $4.5 \mathrm{~cm}$ \\
$\sigma_{P t}$, lab frame & $30.0 \mathrm{MeV} / c$ \\
Longitudinal bunch spread $\sigma_{z}$ & $31.6 \mathrm{~cm}$ \\
Longitudinal emittance $\epsilon_{L n}$ & $849 \mathrm{~mm}$ \\
$6 \mathrm{D}$ invariant emittance & $1200 \mathrm{~mm}$ \\
\hline \hline
\end{tabular}




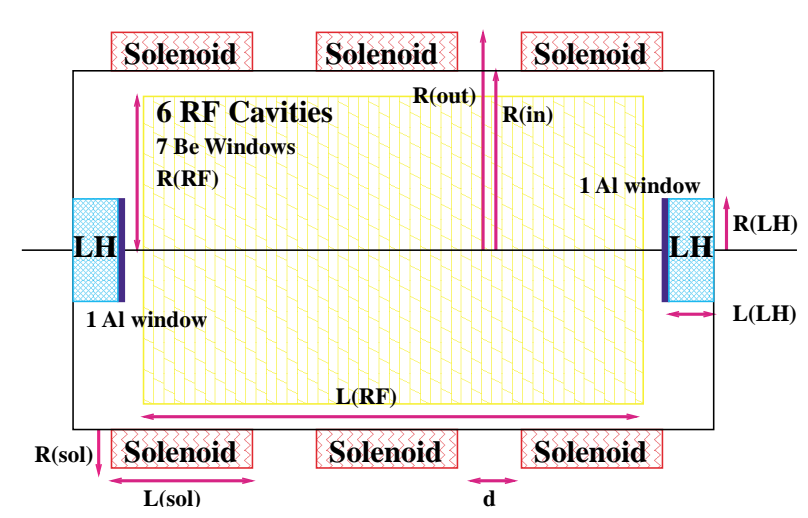

FIG. 8. (Color) Single flip cooling cell layout.

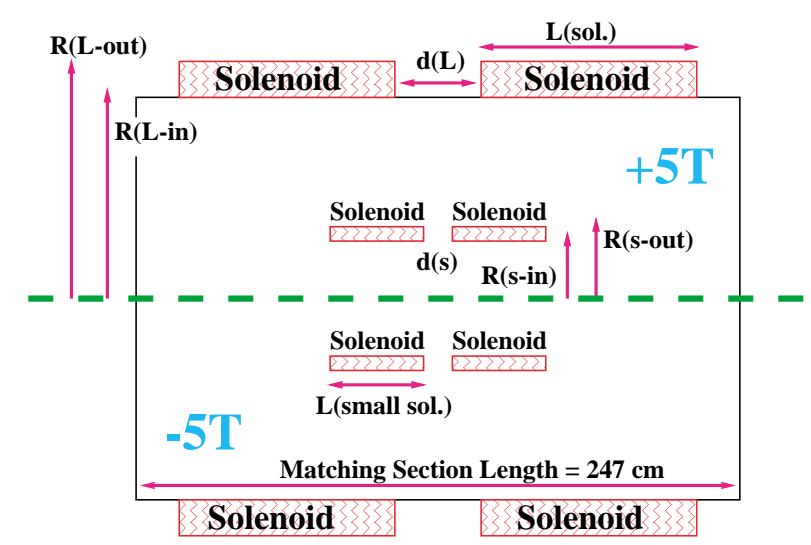

FIG. 9. (Color) Single flip matching section.

Each $2.47 \mathrm{~m}$ cooling cell contains one liquid hydrogen absorber of length between 31 and $34 \mathrm{~cm}$, with radius $20 \mathrm{~cm}$, and one $1.97 \mathrm{~m}$ long linac. The accelerating structure consists of six $\pi / 2$ mode pillbox cavities, with a peak gradient of $15 \mathrm{MV} / \mathrm{m}$, operating at about $30^{\circ}$ synchronous phase. The cells of the linac are separated by $125 \mu \mathrm{m}$ thick beryllium windows, with radius $19 \mathrm{~cm}$. By design, the nominal particle gains $12.5 \mathrm{MV}$ per linac and loses $10 \mathrm{MeV}$ per absorber, thus the nominal channel momentum increases linearly by $2.5 \mathrm{MeV}$ per $2.47 \mathrm{~m}$ long cell. This acceleration is chosen to increase the acceptance of the rf bucket as the rms energy spread increases through the channel, and thereby avoid particle loss due to this longitudinal phase space dilution. To maintain a constant energy loss per cell of $10 \mathrm{MeV}$, the lengths of the absorbers increase from 31.8 to $35 \mathrm{~cm}$. The list of parameters for the $5 \mathrm{~T}$ single flip channel is shown in Table III, and the layouts for the cooling cells and the field flip section are shown in Figs. 8 and 9, respectively.

\section{B. Simulation results}

The single flip cooling channel increases the number of particles within the $6 \mathrm{D}$ acceleration acceptance phase space by a factor of 5 in $150 \mathrm{~m}$. The transmission through the cooling channel is $80 \%$, and approximately half of these particles are within the transverse acceleration acceptance cuts. Nearly all particles transmitted through the cooling channel are within the longitudinal acceleration acceptance.

The minimum achievable emittance is limited by multiple scattering. In this channel, the multiple scattering limit (for an absorber of length $32 \mathrm{~cm}$ ) is $0.0162 \mathrm{rad}$. The initial rms angular spread of the beam $\sigma_{x^{\prime}}=0.1604 \mathrm{rad}$. After 21 sections, $\sigma_{x^{\prime}}=0.0489 \mathrm{rad}$, a reduction by approximately a factor of 3 . The transverse size of the beam, $\sigma_{x}$, is unchanged. The length of the first channel could, in principle, be extended to cool to the multiple scattering limit; however, the $21 \times 2.47 \mathrm{~m}$ length is chosen to both minimize the total length of the cooling channel and compensate for the expansion of the beam envelope in the matching section. In a conservative matching section design, the change in axial field polarity occurs in approximately $0.8 \mathrm{~m}$. The reduction in solenoidal focusing and the large radial fields cause the rms angular spread to increase by about a factor of 3 . The second cooling channel reduces $\sigma_{x^{\prime}}$ by the same factor, and $\sigma_{x}$ by approximately a factor of 2 . The length of the second cooling channel is additionally constrained by the requirement that the beam exits the solenoid with the appropriate transverse correlations, such that the beam outside the solenoid has no angular momentum.

The majority of the particle loss occurs at the field flip, where the momentum spread increases dramatically, due to the radial fields. The longitudinal phase space dilution is particularly evident, as can be seen in Fig. 10. The momentum perturbation at the location of the change in field polarity excites a large synchrotron oscillation, which
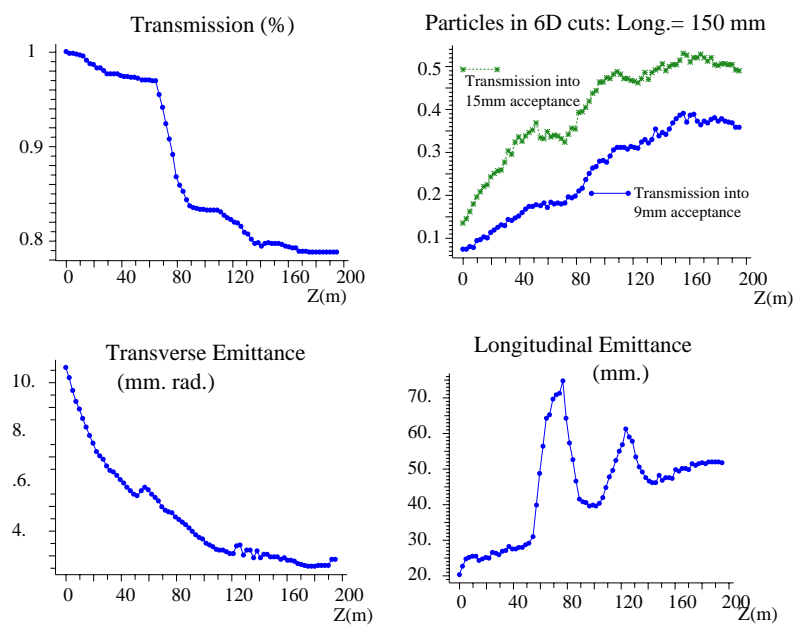

FIG. 10. (Color) Performance of the single flip channel. Top left: fractional transmission; top right: fractional transmission into acceleration acceptance, lower curve (blue) with a $9.375 \mathrm{~mm}$ rad transverse cut, upper curve (green) with a $15 \mathrm{~mm}$ transverse cut; bottom left: transverse emittance; bottom right: longitudinal emittance. 
TABLE IV. Evolution of beam parameters in the single flip cooling channel. The number of muons per proton incident on target is shown in parentheses in the last three rows.

\begin{tabular}{cccc}
\hline \hline Parameter & $z=0 \mathrm{~m}$ & $z=100 \mathrm{~m}$ & $z=150 \mathrm{~m}$ \\
\hline$\epsilon_{T}(\mathrm{~mm} \mathrm{rad})$ & 11.5 & 3.7 & 2.9 \\
$\epsilon_{L}(\mathrm{~mm})$ & 20 & 40 & 47 \\
$N_{9 \mathrm{~mm}}(\%)$ & 7 & 28 & $35(0.077)$ \\
$N_{15 \mathrm{~mm}}(\%)$ & 13 & 46 & $50(0.11)$ \\
$N_{\text {part }}(\%)$ & $100(0.22)$ & $84(0.185)$ & $80(0.176)$ \\
\hline \hline
\end{tabular}

causes particles at high transverse amplitudes to effectively fall out of the rf bucket. By the end of the cooling channel, such large-amplitude particles have been scraped longitudinally, resulting in a net growth of the longitudinal emittance of only a factor of 2.2 , due primarily to straggling. The channel performance is summarized in Table IV, and the transmission and emittance as a function of $z$ are shown in Fig. 10.

\section{CONCLUSION}

We have presented two distinct designs of ionization cooling lattices based on solenoidal focusing elements and liquid hydrogen absorbers. These designs have been optimized for initial beam parameters relevant to an intense neutrino source [15], and engineering feasibility has been used as a guideline to constrain the parameters chosen for the solenoids, the hydrogen absorbers, and the rf systems. The two lattices differ in the type of solenoidal focusing used to minimize $\beta_{\perp}$ at the absorber: the FOFO uses short solenoids (with respect to the Larmor wavelength) with alternating field polarity, while the single flip uses long solenoids, with only one field polarity change. The two lattices have different design limitations: the periodic nature of the FOFO introduces betatron resonances, while the strong perturbation of the beam's transverse motion in the field-flip section of the single flip introduces longitudinal phase dilution (and thus losses) due to the large transverse-longitudinal phase space coupling.

We have shown that with careful optimization of the design parameters these inherent problems are manageable, while respecting the standards of cooling lattice engineering feasibility. Both designs produce a cooling factor of 4.5 for cooling-channel length of $150 \mathrm{~m}$, where cooling factor is defined as the increase in muon intensity within the acceptance of the subsequent acceleration system. This is within $40 \%$ of the cooling goal that has been set for a successful neutrino factory [15]. The two lattices also have similar total muon transmission rates of $70 \%-80 \%$. The performance of ionization cooling lattices of the types discussed in this paper can be further improved by implementing different variations of the solenoid field configuration. For example, in the short alternating solenoid case considered here (FOFO), the magnetic field contains only one harmonic (simple sinusoidal function of position). Other designs, currently under consideration, which use more sophisticated coil configurations may deliver improved cooling performance by introducing more harmonics to the field $[21,22]$.

\section{ACKNOWLEDGMENTS}

This work was supported in part by grants from the Illinois Board of Higher Education, the Illinois Department of Commerce and Community Affairs, the National Science Foundation, and the U.S. Department of Energy.

[1] C. M. Ankenbrandt et al., Phys. Rev. ST Accel. Beams 2, 081001 (1999).

[2] G. I. Budker and A. N. Skrinsky, Sov. Phys. Usp. 21, 277 (1978).

[3] D. Neuffer, Part. Accel. 14, 75 (1983).

[4] G. Penn and J.S. Wurtele, Phys. Rev. Lett. 85, 764-767 (2000).

[5] D. M. Kaplan, Nucl. Instrum. Methods Phys., Res. Sect. A 453, 37 (2000).

[6] E. Kim, C. Kim, G. Penn, A. M. Sessler, and J. S. Wurtele, Fermi National Laboratory Mucool Note No. 36, 1999 (http://www-mucool.fnal.gov/mcnotes/muc0036.ps).

[7] Ya. Derbenev, Fermi National Laboratory Mucool Note No. 108, 2000 (http://www-mucool.fnal.gov/mcnotes/ muc0108.ps); V. Balbekov, in Proceedings of the Neutrino Factory Workshop, 2000 (unpublished).

[8] R. Fernow, in Proceedings of the 1999 Particle Accelerator Conference, New York, edited by A. Luccio and W. MacKay (IEEE, Piscataway, NJ, 1999), p. 3020.

[9] P. Lebrun, see www-pat/simulations/muc; also GEANT3 User Manual (http://wwwinfo.cern.ch/asd/geant/index. html).

[10] Martin Reiser, Theory and Design of Charged Particle Beams (Wiley-Interscience, New York, 1994), Sec. 3.4.4.

[11] Ignoring the MCS term, Eq. (1.1) results in $\epsilon(z)=$ $\boldsymbol{\epsilon}(0) e^{-\left[z /\left(\beta^{2} E d z / d E\right]\right)}=\boldsymbol{\epsilon}(0) e^{-[z /(p d z / d p)]}$, which motivates the definition of $\zeta=\frac{z}{p}\left|\frac{d p}{d z}\right|$ as the scaled longitudinal coordinate.

[12] These expressions result from the evolution of the initial beam distribution (1.4), using Eq. (1.1). See also V. Balbekov, Fermi National Laboratory Mucool Note No. 118, 2000 (http://www-mucool.fnal.gov/mcnotes/muc0118.ps); C. Wang and K. Kim, Fermi National Laboratory Mucool Note No. 92, 2000 (http://www-mucool.fnal.gov/ monotes/muc0092.ps).

[13] C. Wang and K. Kim, Fermi National Laboratory Mucool Note No. 142, 2000 (http://www-mucool.fnal.gov/ monotes/muc0142.ps).

[14] R. Palmer, Fermi National Laboratory Mucool Note No. 46, 1999.

[15] N. Holtkamp and D. Finley (Fermilab Report No. Fermilab-PUB-00-108-E).

[16] J. Miller (private communication).

[17] ASME Boiler and Pressure Vessel Code, ANSI/ASME BPV-VIII-I (American Society of Mechanical Engineers, New York, 1980), part UG-32. 
[18] J. W. Mark, SLAC Report No. SLAC-PUB-3169, 1984.

[19] E. J. Beise et al., Nucl. Instrum. Methods Phys. Res., Sect. A 378, 383 (1996).

[20] A. Moretti, N. Holtkamp, T. Jurgens, Z. Qian, and V. $\mathrm{Wu}$, in Proceedings of the 20th International Linac Conference (Linac 2000), Monterey, California, 2000, eConf C000821:THc18, 2000.
[21] V. Balbekov, Fermi National Laboratory Mucool Note No. 130, 2000 (http://www-mucool.fnal.gov/mcnotes/ muc0130.ps).

[22] B. Palmer, Fermi National Laboratory Mucool Note No. 114, 2000 (http://www-mucool.fnal.gov/mcnotes/ muc0114.ps). 